\title{
Interpersonal Privacy Management in Distributed Collaboration: Situational Characteristics and Interpretive Influences
}

\author{
Sameer Patil ${ }^{1}$, Alfred Kobsa ${ }^{1}$, Ajita John ${ }^{2}$, Lynne S. Brotman ${ }^{2}$, \\ and Doree Seligmann ${ }^{2}$ \\ ${ }^{1}$ Department of Informatics, University of California, Irvine, Irvine CA 92697, USA \\ \{patil, kobsa\} @uci . edu \\ ${ }^{2}$ Collaborative Applications Research, Avaya Labs, Basking Ridge NJ 07920, USA \\ \{ajita, lynne, doree $\}$ @avaya.com
}

\begin{abstract}
To understand how collaborators reconcile the often conflicting needs of awareness and privacy, we studied a large software development project in a multinational corporation involving individuals at sites in the U.S. and India. We present a theoretical framework describing privacy management practices and their determinants that emerged from field visits, interviews, and questionnaire responses. The framework identifies five relevant situational characteristics: issue(s) under consideration, physical place(s) involved in interaction(s), temporal aspects, affordances and limitations presented by technology, and nature of relationships among parties. Each actor, in turn, interprets the situation based on several simultaneous influences: self, team, work site, organization, and cultural environment. This interpretation guides privacy management action(s). Past actions form a feedback loop refining and/or reinforcing the interpretive influences. The framework suggests that effective support for privacy management will require that designers follow a socio-technical approach incorporating a wider scope of situational and interpretive differences.
\end{abstract}

Keywords: Privacy, Awareness, Distributed collaboration, Privacy management.

\section{Introduction}

High-speed networking has enabled organizations to form teams of geographically dispersed knowledge workers. Using such teams offers several advantages: opportunities to employ talent from across the world, possible expansion to distant markets, and savings in operating expenses and travel costs. At the same time, the physical $[1,2]$ and/or temporal [3] separation in such teams poses several challenges when compared with conventional co-located collaboration. A crucial factor that impacts distributed collaborative activities is the impoverishment of interpersonal interactions (professional as well as social) and, consequently, reduced awareness of the activities of others [2, 4]. Awareness information, however, is instrumental for structuring and coordinating one's own work in light of interdependencies [5]. As a remedy, various interpersonal awareness and interaction systems (IAIS) have been built to serve the 
needs of distributed collaborators (see e.g., [5-7]). There is a growing trend in organizations to deploy IAIS to support work activities and interactions of their employees [8-10]. The estimated economic impact of IAIS usage may be to the tune of several billion dollars. IBM alone, for example, estimates more than $\$ 110$ million in annual savings from the use of IAIS [11].

Designers of these systems face the significant challenge of having to attend simultaneously to users' awareness as well as privacy needs [12, 13]. Insufficient attention to either need could undermine the usage of a system. When users cannot effortlessly reconcile privacy desires with awareness needs, they may not utilize the system's full potential [7, 14, 15]. Moreover, insufficient attention to privacy aspects may evoke strong user backlash [16] that can lead to minimal use, or even abandonment, of the system. If this were to happen, organizations stand to lose their investments in IAIS. Moreover, the companies that design and build these systems, as well as their clients, face the prospect of longer-term damage to trust and credibility [17, 18]. In order to build and deploy IAIS that are sensitive to the privacy expectations and behaviors of collaborators, we must first understand how knowledge workers engaged in distributed collaboration currently manage their privacy. This paper reports the findings from a study carried out with this goal.

\section{Related Work}

Prior field studies of geographically distributed teams have been conducted with the primary objective of comparing their practices with those of conventional co-located teams. Therefore, these efforts have attempted to measure efficiency (e.g., [2]), uncover cultural barriers (e.g., [19]), or identify effective resource allocation (e.g., [20]). Our study sets itself apart with its focus on privacy management practices.

Initial findings related to privacy in collaboration were primarily noted "on the side" in studies aimed at evaluating awareness systems. For instance, Dourish [21] characterized privacy controls along a "social-technical continuum." On the social end of this continuum, social pressures and norms are relied upon to prevent system abuse, while on its technical end, technology prevents attempted misuse. Social controls are likely to work well only within small and relatively well-knit communities $[21,22]$. Recently, studies of awareness systems have targeted privacy as the primary object of investigation and unveiled a number of factors that affect users' privacy judgments. These include the user's relationship with the information recipient, the purpose and usage of requested information, the context, and the sensitivity of the content $[15,17,18,23-25]$. In contrast, our study does not limit itself to specific systems or scenarios; we examined privacy management as it occurs in distributed collaboration. We treated IAIS as "part of the circumstance within which those concerns are formulated and interpreted" [26].

Prior research has also generated models to conceptualize privacy in networked multimedia environments. Adams [17] proposed an empirically derived privacy model in which a user's privacy perceptions are based on the sensitivity, receiver, and usage of information. However, her subjects were not seasoned team workers but university students/staff and conference attendees. Palen and Dourish [26] presented a conceptual analysis that describes privacy as a process regulating the boundaries of 
disclosure, identity and temporality. This process is both dynamic (i.e., shaped by personal and collective experiences and expectations) and dialectic (i.e., under continuous boundary negotiation). Lederer et al. [27] further listed system properties, actor relations, and information types as relevant interrelated dimensions that are important for the privacy management process. The framework we describe in this paper supports and extends these insights by grounding them in an empirical study of geographically distributed collaborators.

\section{Study Setting}

We studied a software development project (henceforth Project X) at a large multinational telecommunications company. At various stages, Project X included anywhere between 80 to 130 contributors spread across at least five different locations: four in the U.S. (in three different time zones) and one in India. The software developed by Project X comprised eighteen interdependent modules integrated into a single release. Project X releases were arranged in cycles of three to four months. Each new release incorporated new features and fixed bugs from the previous releases. During each cycle, the management team of Project $\mathrm{X}$ tracked the progress of the ongoing cycle and planned out the details of the next cycle.

A variety of contributors worked on Project X: managers at different hierarchical levels, software architects, systems engineers, software developers, testers, source code management (SCM) support staff, and internal and external customers. The number of people at each location varied from a handful to more than thirty. It should be noted that team ${ }^{1}$ membership was independent of geographical location. In other words, it was not uncommon for members of a team to be distributed across different sites. In addition, a limited amount of telecommuting was common, but only in the U.S. Several forms of telecommuting were practiced: telecommuting for part of the day, telecommuting for the entire day, telecommuting on an as-needed basis etc. In a couple of cases, project members worked mostly from their "virtual office" at home, coming in to the workplace only as needed.

Project X required frequent intra-module as well as inter-module collaboration in all phases: design, architecture, coding, testing, integration, and maintenance. Collaboration was also required when the employees responsible for a module changed; the new individual(s) needed to be briefed. Some collaborative activities spanned the entire project scope (for example, when management planned project activities, tracked progress, made adjustments, and set strategic goals using estimates and forecasts). The SCM unit provided services to Project X for managing its source code repositories. Representatives from other software projects that would use the Project $\mathrm{X}$ platform were also consulted on requirements and feedback. For collaboration, Project X members used not only face-to-face meetings but also a variety of technical communication, coordination and awareness tools. These included email, Instant Messaging (IM), shared calendars, Microsoft Project ${ }^{\circledR}$, telephones (desk phones as well as mobile phones), the SCM system, shared document repositories, and various Internet-based conferencing tools (such as Virtual Network Computing (VNC) or Microsoft NetMeeting ${ }^{\circledR}$ ).

\footnotetext{
${ }^{1}$ We loosely define a team to be a group of individuals supervised by the same manager.
} 
While most developers, testers and lower-level managers worked solely on Project $\mathrm{X}$, other contributors were engaged in multiple projects. The fraction of time they spent on Project X ranged from 3-4\% to more than 50\% and was partly dependent on the current stage in the release cycle. For instance, in some stages a systems engineer would spend nearly all of his or her work time on Project X, while during the rest of the cycle, his or her effort would be an order of magnitude lower.

\section{Methodology}

Our research objective was to investigate how geographically distributed collaborators reconcile the often conflicting needs of awareness and privacy. To avoid biasing the participants, the advertised goal of the study was to investigate collaborative work practices. We did not impose a definition of privacy on the study participants. Rather, we asked them to explain what "privacy" meant to them in the context of their work and work practices. Given the lack of a universal definition for privacy, our intention was to avoid biasing the participants or confining them to a specific view of privacy. Instead, we sought to uncover the various contextual meanings of privacy for the participants, and the associated behaviors and practices aimed at satisfying privacy needs. An initial hour-long conversation with the Head of Project X helped us gain a basic understanding of the project and formulate a plan for conducting the study. Thereafter, we used the following methods:

- Non-participant Observation. Our exploration started with non-participant observation of the meetings of the Project X management team. These meetings, which involved managers from all project sites and all hierarchical levels, were used to formulate the detailed plan for the next release cycle of the project. These observations improved our understanding of the organization and activities of the project, which in turn guided the development of the other aspects of the study that are described below.

- Site Visits. During the first phase of the study, the researcher was based at a Project $\mathrm{X}$ site on the East Coast of the U.S., and visited three of the other sites, in mid-U.S., on the U.S. West Coast, and in India, each for about a week. At every location, interviews were conducted with project members (see below), and site-specific factors such as architecture, layout, work practices and culture were documented. We also interviewed project contributors from the remaining U.S. site - also located on the East Coast - while they were visiting the first-mentioned site.

- Semi-structured Interviews. At each visited site, we conducted semi-structured interviews lasting about 90 minutes. The questions were divided into three main themes: work practices, awareness and privacy needs, and desired enhancements to collaborative tools. In total, we interviewed fifty-two project members across the five sites. Interviewees were chosen in such a way that the different job functions at the various sites were covered to the extent possible.

- Online Questionnaire. Based on key insights from the above activities, we formulated an online questionnaire. It aimed at probing deeper into some of the aspects we learned from the earlier activities and at attaining broader coverage across Project $\mathrm{X}$ by reaching those whom we had not been able to interview. Additionally, we used questions from the literature to measure privacy attitudes and practices in the 
domain of consumer privacy [28, 29], and collected demographic information. The questionnaire was distributed to all individuals involved with Project $\mathrm{X}$ at that time (roughly 125). We obtained 90 valid responses (response rate of 74\%) which included responses from 30 of our original 52 interviewees.

Transcripts of the interviews were analyzed using the grounded theory approach [30]. Categories that emerged from open coding were further refined into ten higher-level categories by selective coding. Then, axial coding was employed to identify the relationships between these higher-level characteristics resulting in a framework that illustrates how privacy management operates in the collaborative work context.

\section{Privacy Management Framework}

Fig. 1 shows the framework that emerged from our analysis. It presents privacy management practices in collaborative work as dependent not just on a number of situational characteristics but also on a hierarchy of personal interpretive influences that an individual applies to the situation. As can also be seen in Fig. 1, the interpretation applied by an individual to the situation at hand leads to privacy management action(s), or lack thereof. Moreover, these actions themselves form a feedback loop that contributes to the reinforcement and/or evolution of the interpretive influences over time. Privacy is known to be a context-dependent and highly personal concept [26, 31]. The framework captures the former aspect in terms of the situational characteristics, and the latter through the interpretive influences. (The interpretive influences are related to the "identity" boundary whereas the situational characteristics encompass the "disclosure" and "temporal" boundaries described by Palen and Dourish [26].)

\subsection{Situational Characteristics}

Our analysis revealed five key situational characteristics that interviewees deemed important when reconciling awareness and privacy needs.

- Issues. The details of the issue(s) at hand were instrumental in judging aspects such as confidentiality, urgency, audience, or communication medium. These judgments, in turn, affected privacy management actions.

Issue(s) under consideration

Affordances of technology

Physical space

Temporal aspects

Relationships with parties

Situational Characteristics

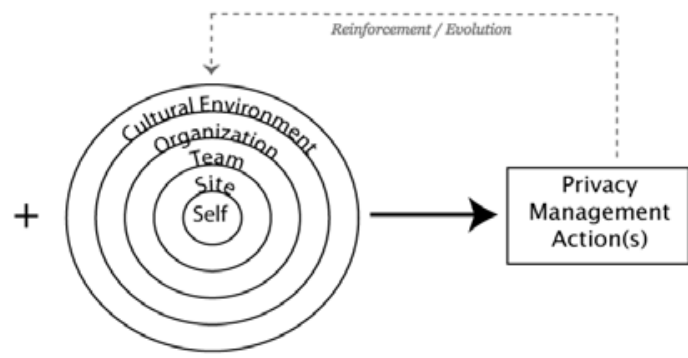

Interpretive Influences

Fig. 1. Privacy management described in terms of interpretive influences applied to situational characteristics 
- Relationships. This characteristic refers to the nature of relationships - formal as well as informal - that existed among the various parties involved in a particular situation.

- Temporality. Two temporal aspects impacted a situation. The first was the time of day in one's own time zone as well as in those of one's collaborators. The second was the temporal extension of the present action(s) into the future in the form of archives, logs, records, or people's memories.

- Technology. As described in Section 3, collaborative activities utilized a host of technologies. The affordances as well as the limitations of a system constrained which actions it could support, and in what manner [32].

- Space. This characteristic refers to the physical space in which work was carried out. It includes the design and layout of workspaces and work sites, and also of other locations from which individuals worked (such as homes, conference rooms, offices of others, cars, and hotels).

The situational characteristics Issues, Relationships and Technology map to and refine, respectively, the concepts System Properties, Actor Relations, and Information Types proposed by Lederer et al. [27]. Similarly, Issues, Relationships and Temporality subsume Information Sensitivity, Receiver and Usage from Adams' model [17]. Even though we discussed each of the characteristics separately above, a multiple of these often came into play in any given situation. In other words, all of them were subject to interpretation simultaneously. This is illustrated by the supporting examples in Section 6.

\subsection{Interpretive Influences}

While the characteristics described above set the stage, the privacy management action(s) $)^{2}$ of each individual further depended on his or her interpretation of the situation. We identified five major influences that guided this interpretation.

- Self. Individuals drew upon their personal disposition and characteristics when interpreting a situation.

- Team. The practices, norms or policies of one's team were also crucial in deciding how situations were interpreted. We observed that the impact of this influence was dependent on factors such as the length of time the team members had worked together, the degree of work coupling, and the management style of the team leader.

- Site. This influence refers to practices and local factors that were unique to a given site. For example, the typical practice at the U.S. West Coast site was to arrive at the workplace later in the morning than at the other U.S. sites.

- Organization. The multi-national corporation was the umbrella uniting the different sites. It influenced interpretation by providing policies and norms, a shared sense of identity, as well as a shared technical infrastructure for carrying out work activities.

- Cultural Environment. The cultural environment external to the organization in which one was embedded also influenced how situations were interpreted. The large differences between the privacy preferences and practices at the India site

\footnotetext{
${ }^{2}$ The framework also treats inaction, i.e., deciding not to act, as an action.
} 
compared with those at the U.S. sites is one of the salient findings of the study (described in [33]). These differences were partially attributed to the impact of the cultural environment.

We also noted that the above influences could be arranged in a hierarchy beginning with the most inward influence (self), and growing progressively outward toward the larger environment one is embedded in. The interrelationship between the influences also needs to be emphasized. For instances, differences between sites can be attributed not just to local factors (such as the history of the site, the interactions among local colleagues, the weather etc.) but also to organizational factors (such as policies or infrastructural variations) as well as cultural influences. In order to isolate the contribution of an individual influence, it may be necessary to make comparisons. For example, the cultural environment is unlikely to be a major contributor to differences among sites within the U.S.

\section{Supporting Examples}

In this section, we present four frequently encountered situations in which collaborators are faced with reconciling awareness and privacy needs: making communication ${ }^{3}$ choices, handling interruptions, working from home, and dealing with urgent matters. The following subsections describe how the framework presented above explains the privacy management actions that we encountered in these situations (the labels in italics indicate the applicable situational characteristics and interpretive influences). Although we discuss the four separately, it should be noted they are often interrelated. For instance, communication choices may need to be made when handling interruptions, or an interruption may need to be handled to deal with an urgent situation.

\subsection{Making Communication Choices}

Our interviewees indicated that privacy concerns impacted their communication choices, i.e., what was communicated to whom and how. Several privacy management practices occurred in the context of communication, such as self-censorship, medium switching, location switching, etc. When engaging in these practices, interviewees reported taking into account the situational characteristics outlined in our framework. Thus, privacy management actions depended on factors such as the importance, sensitivity, and confidentiality of the matter being communicated (issues), hierarchical as well as social relationships with the audience (relationships), temporal considerations such as whether or not the communication could be archived and accessed at future times (temporality), the richness of expression afforded by a communication technology (technology), and the presence of others around oneself who may come to know about the communication (space).

\footnotetext{
3 "Communication" refers not just to the contents of verbal or written conversations, but also to other more implicit interaction aspects, such as IM status, calendar entries, or code submission time stamps, which can serve to communicate awareness information about actions, availability, etc.
} 
Commensurate with our framework (see Fig. 1), the link between a particular communication situation and the corresponding privacy management actions is established by the interpretive influences. For instance, some individuals preferred IM over email for short messages (self, issues). Interviewees also reported that their communication choices were influenced by the norms in the team, and the management style of their manager. For example, some participants reported backchannel IM conversations with other team members in order to present a "uniform voice" during meetings with others, while others reported being available by mobile phone at all times because of managerial expectations (team). Site-specific influences were also observed: due to cubicle environments at some of the sites, private phone conversations necessitated reserving conference rooms or stepping outside the building (site). General organization-wide influences such as the shared technical infrastructure, and corresponding communication norms (e.g., on sharing one's calendar or accessing the calendars of other employees), also shaped how privacy was managed in communication (organization). Moreover, external factors unrelated to work, such as family, commuting conditions, and the cultural background, influenced how situations were interpreted (cultural environment).

Interviewees expressed vastly different expectations of privacy for written vs. nonwritten communication (technology). Written communication (which included email) was composed with care, and was often self-censored (issues, relationships, self, organization). The fact that it could be saved, or be forwarded beyond the original recipient, was often taken into account (temporality). The choices of whom to copy, or leave out, were made deliberately (issue, relationships). Interviewees also applied their interpretations to the variety of non-communicative functions of written communication, such as its role as an individual memory aid, knowledge management archive, organizational record, and instrument of accountability. On the other hand, non-written communication (such as phone calls and face-to-face meetings) was more informal and impromptu (relationship, technology, team, site). In terms of privacy, it was sometimes used for discussions deemed too sensitive for the written medium and/or when the individual wished to avoid a written trail (issue, technology, temporality). IM fell somewhere in between [34]. Although IM is written communication, a majority of our interviewees treated it as ephemeral and informal. With the exception of a few, who exercised the same caution with IM as with email, the interviewees did not report archiving IMs (in fact, some even claimed not to know that it was possible to save IMs), and assumed that other employees did not save IMs either (technology, temporality, organization).

\subsection{Handling Interruptions}

One characterization of privacy is "freedom to be left alone" [35], i.e. control over access to oneself. Interruptions, which are common in knowledge work [36], have a direct impact in this regard [37]. Our interviewees mentioned several kinds of interruptions, planned as well as unplanned. These included: scheduled meetings (temporality), incoming communication (e.g., email, IM, phone) (technology), colleagues dropping by one's office (relationships), urgent issues that required immediate attention (issues, temporality), and lunch breaks (temporality). We noted several privacy management 


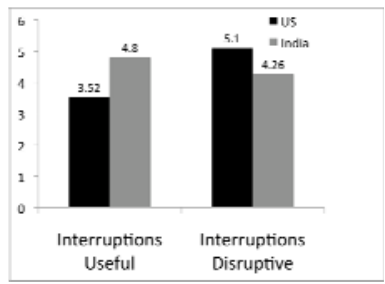

Fig. 2. Contrasting attitudes towards interruptions at the U.S. and India sites

practices for dealing with interruptions: closing the office door (space), scheduling "busy" blocks on one's calendar (temporality, technology), turning off IM or setting the IM status to "busy" (technology), multitasking during conference calls (temporality, technology), eating lunch at one's desk (temporality, space), working from home (space), and working during hours when few others are present (temporality).

Interruptions exhibited disruptive as well as useful characteristics. On the one hand, interviewees complained that interruptions took attention away from their current tasks (issues), required extra time and effort for refocusing on the original task (issues, temporality), and split time into short blocks resulting in the "filler" blocks being spent unproductively (temporality). On the other hand, interviewees recognized the value of interruptions for learning about issues that required immediate attention (issues), coordinating with colleagues (issues, relationships, temporality), taking a break (temporality), multi-tasking (issues, temporality), interacting with friends and colleagues at the workplace (regarding work as well as non-work matters) (relationships), and attending to domestic errands (issues, space).

In handling interruptions, privacy management involved applying the interpretive influences to resolve the tension between the disruptive influences of interruptions with their potential usefulness. (Note that merely "mentally processing" an interruption is also disruptive even if the interruption is not dealt with immediately.) For instance, we found that the job function affected preferences regarding how the tension ought to be resolved (self). For example, managers recognized greater value in interruptions since managerial duties require them to be available to resolve the issues brought forth by their subordinates [38]. In contrast, developers desired long, uninterrupted time blocks to concentrate on their programming tasks. Based on responses to the online questionnaire, we also uncovered differences between those at the U.S. sites with those in India (space, site, cultural environment) [33]. As Fig. 2 shows, those in India agreed more than those in the U.S. that interruptions are useful (India: 4.80, US: 3.52, $\mathrm{p}<0.0001)$. In contrast, workers at the U.S. sites found interruptions disruptive to a larger extent (India: 4.26 US: $5.10 \mathrm{p}<0.015$ ).

\subsection{Balancing Work and Home}

As mentioned in Section 3, it was not uncommon for Project $\mathrm{X}$ members to work from home, at least in the U.S. ${ }^{4}$ The form of telecommuting ranged from occasionally

\footnotetext{
${ }^{4}$ Telecommuting was practically non-existent in India due to the lack of adequate domestic technical infrastructure, and demographic and cultural differences (cultural environment).
} 
checking email from home to having a permanent home office (temporality, space). Most of our interviewees fell somewhere between these two extremes (see Section 3). Moreover, more than half of our interviewees worked from home during late evenings and/or early mornings (temporality). This was necessitated by the need to "get work done" in long uninterrupted time blocks (issues, temporality), to "catch up" with unfinished tasks (issues, temporality), to handle urgent matters (issues, temporality), to interact with people in other time zones (issues, relationships, temporality, technology), and to build new knowledge and skills. Similarly, several interviewees admitted attending to domestic tasks at the workplace (issues, temporality, space). Examples include errands, doctor's appointments, personal email/IM/Web activities, family phone calls, or childcare tasks ${ }^{5}$. Traditionally, the two primary spheres of an individual's life - domestic and professional - have been markedly distinct. For Project X workers, however, the possibilities of getting work done even when away from the office blurred the boundary between home and work, and consequently impacted the traditional notion of the 9-to-5 work day (temporality, space). A second factor impacting the notion was the time zone differences with one's collaborators (temporality). As a result, what was considered to be "typical" work hours varied from location to location (site) and person to person (self).

Privacy expectations at home and at work are different. Accordingly, we noted several privacy management practices to balance work and home based on one's interpretation of the telecommuting situation. Outside of the standard business hours, almost all interviewees desired and exercised more control over their availability to others (issues, relationships) and over the tasks they worked on (issues). For example, during non-business hours some interviewees chose not to sign-in to IM (technology), or chose to work on tasks that did not require interaction with others (issues). In many cases, such preferences (self) applied regardless of one's physical location during these times, i.e., regardless of whether one was working at home or from one's workplace (space). For instance, frequent telecommuters reported that during standard business hours, they strived to make themselves available to their collaborators to the same extent that they would at the workplace (temporality, space, team). Most interviewees also reported designating a separate room or work space at home (space), and trying to separate work and home activities by using separate computers and phones (issues, technology).

\subsection{Dealing with Urgent Matters}

While all the situations discussed above pertain to normal routines, dealing with urgent matters (issues, temporality) required deviating from typical privacy expectations and practices. Judgments of urgency were based not only on the matter at hand but also on the parties involved (relationships). For instance, a request from one's boss was often assigned higher urgency. Discussions of urgency came up frequently in our interviews; participants stated that "normal" privacy expectations and practices did not apply in urgent situations. In such cases, they engaged in privacy management practices different from their normal preferences (self): reorganizing their calendar to

${ }^{5}$ Interestingly, the India site employed a person who was charged solely with attending to the domestic errands of the knowledge workers (e.g., paying bills at different places in town) (cultural environment). 
devote time to "put out the fire" (issues, temporality), limiting their availability for other tasks (issues, temporality), making themselves reachable on their mobile phones (temporality, space, technology), answering phone calls at odd hours (temporality, space, technology), and ensuring their availability even on vacation (temporality, space). Others were expected to make similar adjustments to their privacy expectations. Thus, when urgent matters arose, our interviewees interrupted others (issues), preferred a phone call over an email (issues, technology, temporality), called the mobile phones of their collaborators (issues, technology, temporality), or contacted higher-level managers with whom they would not normally communicate (issues, relationships).

In general, the views of all stakeholders (team, site, organization) on what should be regarded as urgent were aligned, but not always. The interviewees reported a handful of instances of differing interpretations of urgency. Since urgency led to changed privacy expectations and practices, mismatches in urgency evaluations could pose problems (such as receiving a phone call in the middle of the night for an issue that one does not deem equally urgent as the caller).

\section{Implications and Future Work}

It is often argued that (a) employees of an organization ought not desire privacy at the workplace, in the interest of organizational needs for transparency and accountability of employee actions, and (b) employees would not harbor privacy concerns from colleagues due to familiarity and shared organizational bonds. In fact, some of our interviewees also expressed similar views initially because they began by characterizing privacy in terms of monitoring by the organization, preventing the disclosure of personal information unrelated to work, or hiding inappropriate activities from the organization and colleagues. Perhaps this resulted from media coverage in other contexts that also spur privacy concerns (e.g., government surveillance, commercial data mining, or personally identifiable data usage by entities such as vendors, companies, universities, hospitals, and governments). In contrast, as the above discussion illustrates, privacy considerations in collaborative work are focused on interpersonal relationships and interactions ${ }^{6}$. Our findings demonstrate that knowledge workers do engage in privacy management, and underscore the need for IAIS that elevate the effectiveness and efficiency with which privacy desires can be reconciled with conflicting collaborative needs.

Users of IAIS use multiple interaction channels; they work in spaces shared with other workers; they multitask; they interact with people with whom they have diverse kinds of relationships; they move between work and home spheres; they need to prioritize their tasks and availability. Our study shows that these situational characteristics are all considered (and usually several at the same time) when managing privacy. Therefore, in order to enhance the consideration of privacy needs, IAIS designers will need to take situational characteristics into account. Improvements to IAIS need not be complex nor purely technical. For example, technical enhancements could be

\footnotetext{
${ }^{6}$ The interviewees and survey respondents attached low significance to the issue of "employer monitoring" of their activities in an institutional sense. Many were, however, significantly more concerned about "managerial monitoring."
} 
augmented by simple additions such as privacy screens for monitors, or dual monitor setups that allow one to channel interruptions to a secondary screen. It may even be beneficial to co-design organizational policies regarding interpersonal privacy expectations and practices along with the technological developments.

In earlier work [15, 39], we established "impression management" [40] as an underlying cause of privacy concerns. We uncovered impression management practices in the present study as well. A number of our interviewees expressed the desire to "appear professional," "present an appropriate image," "avoid being perceived negatively," and "tailor the message to the audience." We plan a deeper exploration of how to translate impression management considerations into design suggestions for improving privacy management.

\section{Conclusion}

We reported on a study aimed at understanding the interpersonal privacy management practices of geographically distributed collaborators. A framework that describes these practices emerged from a grounded theory analysis of interviews with members of a geographically distributed project, coupled with qualitative field observations at the project sites. The framework identifies five key situational characteristics (issues, relationships, temporality, technology, and space) which collaborators interpret based on five key influences (self, team, site, organization, and cultural environment). These interpretations guide privacy management practices, which in turn provide feedback for the reinforcement or evolution of the interpretive influences. These privacy management practices often require weighing multiple situational characteristics under various simultaneous interpretive influences. We therefore believe that in order to facilitate more effective and efficient privacy management, collaborative systems ought to allow a broader consideration of situational characteristics and a greater differentiation of interpretive influences than is currently the case. Our findings highlight the deep embedding of collaborative technical systems as part of the situational context. To build privacy management solutions, it is therefore necessary to follow a socio-technical design approach that encompasses aspects such as the physical space in which IAIS are embedded, and the diversity of relationships between the parties whose interactions they mediate.

Acknowledgments. We thank the study participants for their time. We also acknowledge Mihir Mahajan, Xinru Page and Joel Ossher for their comments. This research has been supported by NSF Grant Nos. 0205724 and 0808783.

\section{References}

1. Olson, G.M., Olson, J.S.: Distance Matters. Human-Computer Interaction 15(2/3), 139178 (2000)

2. Herbsleb, J.D., Mockus, A., Finholt, T.A., Grinter, R.E.: An Empirical Study of Global Software Development: Distance and Speed. In: Proc. of ICSE 2001, Toronto, Canada, pp. 81-90. IEEE Press, Los Alamitos (2001) 
3. Pankoke-Babatz, U., Syri, A.: Collaborative Workspace for Time Deferred Electronic Cooperation. In: Proc. of GROUP 2003, Sanibel Island, FL, USA, pp. 187-196. ACM Press, New York (1997)

4. Kraut, R., Egido, C., Galegher, J.: Patterns of Contact and Communication in Scientific Research Collaboration. In: Proc. of CSCW 1988, Portland, OR, USA, pp. 1-12. ACM Press, New York (1988)

5. Dourish, P., Bly, S.: Portholes: Supporting Awareness in a Distributed Work Group. In: Proc. of CHI 1992, Monterey, CA, USA, pp. 541-547. ACM Press, New York (1992)

6. Cheng, L.T., Hupfer, S., Ross, S., Patterson, J.: Jazzing up Eclipse with Collaborative Tools. In: Proc. of Eclipse 2003, Anaheim, CA, USA, pp. 45-49. ACM Press, New York (2003)

7. Herbsleb, J.D., Atkins, D.L., Boyer, D.G., Handel, M., Finholt, T.A.: Introducing Instant Messaging And Chat In The Workplace. In: Proc. of CHI 2002,, Minneapolis, MN, USA, pp. 171-178. ACM Press, New York (2002)

8. DiMicco, J., Millen, D.R., Geyer, W., Dugan, C., Brownholtz, B., Muller, M.: Motivations for Social Networking at Work. In: Proc. of CSCW 2008, San Diego, CA, USA, pp. $711-$ 720. ACM Press, New York (2008)

9. FaceTime Communications: The Collaborative Internet: Usage Trends, End User Attitudes and IT Impact (2008)

10. Muller, M.J., Raven, M.E., Kogan, S., Millen, D.R., Carey, K.: Introducing Chat into Business Organizations: Toward an Instant Messaging Maturity Model. In: Proc. of GROUP 2003, Sanibel Island, FL, USA, pp. 50-57. ACM Press, New York (2003)

11. IBM: IBM Reaps Business Benefits and Major Cost Savings From Unified Communications and Collaboration (2008)

12. Hudson, S.E., Smith, I.: Techniques for Addressing Fundamental Privacy and Disruption Tradeoffs in Awareness Support Systems. In: Proc. of CSCW 1996, Boston, MA, USA, pp. 248-257. ACM Press, New York (1996)

13. Patil, S., Kobsa, A.: Privacy Considerations in Awareness Systems: Designing with Privacy in Mind. In: Markopoulos, P., de Ruyter, B., Mackay, W. (eds.) Awareness Systems: Advances in Theory, Methodology and Design. Springer, Heidelberg (2009)

14. Lee, A., Girgensohn, A., Schlueter, K.: NYNEX Portholes: Initial User Reactions and Redesign Implications. In: Proc. of GROUP 1997, Phoenix, AZ, pp. 385-394. ACM Press, New York (1997)

15. Patil, S., Kobsa, A.: Instant Messaging and Privacy. In: Proc. of HCI 2004, Leeds, UK, pp. 85-88 (2004),

http: / / www. ics.uci.edu/ kobsa/papers / 2004 -HCI-kobsa.pdf

16. Calore, M.: Privacy Fears Shock Facebook. Wired News (September 2006), http: / / www.wired.com/science/discoveries/news/2006/09/71739

17. Adams, A.: Users' Perception of Privacy in Multimedia Communication. In: Proc. of CHI 1999: Extended Abstracts, Pittsburgh, PA, USA, pp. 53-54. ACM Press, New York (1999)

18. Adams, A., Sasse, M.A.: Privacy Issues in Ubiquitous Multimedia Environments: Wake Sleeping Dogs, or Let Them Lie? In: Proc. of Interact 1999, Edinburgh, UK, pp. 214-221 (1999)

19. Damian, D.E., Zowghi, D.: An Insight into the Interplay between Culture, Conflict and Distance in Globally Distributed Requirements Negotiations. In: Proc. of HICSS 2003, vol. 1, p. 19c (2003)

20. Battin, R., Crocker, R., Kreidler, J., Subramanian, K.: Leveraging Resources in Global Software Development. IEEE Software 18(2), 70-77 (2001) 
21. Dourish, P.: Culture and Control in a Media Space. In: Proc. of ECSCW 1993, Norwell, MA, USA, pp. 125-137. Kluwer Academic Publishers, Dordrecht (1993)

22. Ackerman, M.S., Starr, B., Hindus, D., Mainwaring, S.D.: Hanging on the 'Wire: A Field Study of an Audio-only Media Space. ACM Trans. Information Systems 4(1), 39-66 (1997)

23. Lederer, S., Mankoff, J., Dey, A.K.: Who Wants to Know What When? Privacy Preference Determinants in Ubiquitous Computing. In: Proc. of CHI 2003, Fort Lauderdale, FL, USA, pp. 724-725. ACM Press, New York (2003)

24. Consolvo, S., Smith, I.E., Matthews, T., LaMarca, A., Tabert, J., Powledge, P.: Location Disclosure to Social Relations: Why, When, \& What People Want To Share. In: Proc. of CHI 2005, Portland, OR, USA, pp. 81-90. ACM Press, New York (2005)

25. Olson, J.S., Grudin, J., Horvitz, E.: A Study of Preferences for Sharing and Privacy. In: Proc. of CHI 2005, Portland, OR, USA, pp. 1985-1988. ACM Press, New York (2005)

26. Palen, L., Dourish, P.: Unpacking "Privacy" for a Networked World. In: Proc. of CHI 2003, Fort Lauderdale, FL, USA, pp. 129-136. ACM Press, New York (2003)

27. Lederer, S., Mankoff, J., Dey, A.K.: Towards a Deconstruction of the Privacy Space. In: Ubicomp 2003 Workshop on Ubicomp Communities: Privacy as Boundary Negotiation (2003)

28. IBM: IBM Multi-National Consumer Privacy Survey (October 1999)

29. Westin, A.F.: Harris-Equifax Consumer Privacy Survey 1991 (1991)

30. Glaser, B.G., Strauss, A.L.: The Discovery of Grounded Theory: Strategies for Qualitative Research, 8th edn. Aldine Transaction, Chicago (1967)

31. Acquisti, A., Grossklags, J.: Privacy and Rationality. In: Strandburg, K., Raicu, D.S. (eds.) Privacy and Technologies of Identity: A Cross-Disciplinary Conversation, pp. 15-29. Sprinter, New York (2006)

32. Norman, D.A.: The Design of Everyday Things, 3rd edn. MIT Press, Cambridge (1998)

33. Patil, S., Kobsa, A., John, A., Brotman, L.S., Seligmann, D.: Comparing Privacy Attitudes of Knowledge Workers in India and the U.S (forthcoming, 2009)

34. Voida, A., Newstetter, W.C., Mynatt, E.D.: When Conventions Collide: The Tensions of Instant Messaging Attributed. In: Proc. of CHI 2002, Minneapolis, MN, USA, pp. 187194. ACM Press, New York (2002)

35. Warren, S.D., Brandeis, L.D.: The Right to Privacy. Harvard Law Review 4(5), 193-220 (1890)

36. González, V.M., Mark, G.: Constant, Constant, Multi-tasking Craziness": Managing Multiple Working Spheres. In: Proc. of CHI 2004, Vienna, Austria, pp. 113-120. ACM Press, New York (2004)

37. Altman, I.: The Environment and Social Behavior: Privacy, Personal Space, Territory, Crowding. Brooks/Cole, Monterey (1975)

38. Hudson, J.M., Christensen, J., Kellogg, W.A., Erickson, T.: I'd be overwhelmed, but it's just one more thing to do: Availability and Interruption in Research Management. In: Proc. of CHI 2002, Minneapolis, MN, USA, pp. 97-104. ACM Press, New York (2002)

39. Kobsa, A., Patil, S., Meyer, B.: Privacy in Instant Messaging; An Impression Management Model (forthcoming, 2009)

40. Goffman, E.: The Presentation of Self in Everyday Life. Doubleday, Garden City (1959) 